\title{
Método afectivo-cognitivo para el aprendizaje "MACPA"*
}

\section{The Affective-Cognitive Learning Method (MACPA)}

Gladys Molano Caro**

Corporación Universitaria Iberoamericana gmolano_caro@yahoo.com

Recibido: 26 de noviembre de 2012

Aceptado: 2 de diciembre de 2012

\section{Resumen}

A través de este escrito se da cuenta de un método de enseñanza que fue construido en el diario hacer, con los estudiantes que eran reportados por los docentes porque no aprendían a leer y a escribir de manera oportuna. Aunque sintetiza una opción novedosa' para enseñar a los niños, niñas y jóvenes a leer y a escribir, también se constituye en un método para aquellos estudiantes que se encuentran en un "limbo cognitivo" por presentar una dificultad en el aprendi- zaje para acceder a estas áreas fundamentales para su proceso de formación.

\section{Palabras clave}

Método, lectura, escritura, problemas de aprendizaje.

\section{Abstract}

This paper explores a teaching method that was conceived through the daily interaction with stu-

* Inicialmente MACPA fue construido para los niños que presentan un Problema de Aprendizaje Moderado o Grave, y no encuentran solución al "limbo cognitivo" por el que tienen que transitar; luego se transformó en un método para el aprendizaje de la lectura y la escritura. Debo agregar que a todos los niños de la Institución Educativa Distrital Alemania Solidaria, que de una u otra manera fueron exponiendo el camino para su construcción, les guardo mi más grande sentimiento de gratitud y de afecto.

** La construcción del método fue consecuencia de una investigación que realizó la autora en la Universidad Iberoamericana. En ella participó un grupo de estudiantes de los últimos semestres de la carrera de educación, mereciendo especial recordación personas como Yenny Mora, Edirney Granda, Cristina Rozo. Las demás personas que no aparecen aquí, es porque el espacio disponible no lo hace posible, sin embargo, conservo hacia ellos una especial gratitud.

1 Novedosa porque fue premiada en el año 2009 por el Instituto para la Investigación Educativa, IDEP. 
dents who were documented by their teachers for not being able to read and write in a timely manner. Although it synthesizes a new way of teaching children and young people to read and write, it also becomes a method for students who are in a "cognitive limbo",

\section{Introducción}

La autora del denominado "Método Afectivo-Cognitivo para el Aprendizaje” (MACPA) - quien esto escribe- quiere poner a consideración de los diferentes profesionales que laboran en el campo de la educación, este método que no solo está cimentado en los dispositivos básicos de aprendizaje, sino que tiene en cuenta a la población que evidencia dificultades de aprendizaje, incorporando las características fundamentales de los niños, niñas y jóvenes que los presentan. Para su elaboración también se tuvo en cuenta las diversas situaciones afectivas/ emocionales que están presentes en las historias de vida de los niños, para así seleccionar los diferentes aspectos y referentes que acompañan las tres cartillas que se han elaborado, utilizando los principios que acompañan la propuesta.

En la construcción del método se tuvo en cuenta el conocimiento de la historia del niño en sus aspectos de desarrollo, de experiencias vividas, de las relaciones familiares y académicas. También se incorporó la afectividad, tomada desde dos referentes diferentes como son:

a) El afecto que despliega el maestro hacia el niño, que surge como consecuencia de la comprensión que va teniendo del "limbo cognitivo" por el que transitan los niños con esta condición y;

b) El vínculo afectivo/emocional que evoca o va desarrollando el niño o la niña a partir del conocimiento que tiene o que va incorporando de los diferentes aspectos, situaciones, objetos y personas con las cuales se ha relacio- who show a learning disability in grasping these key subjects for their educational process.

\section{Keywords}

Method, reading, writing, learning disabilities.

nado o se relacionará a medida que va accediendo a las fichas-guías que trae el método.

Asimismo, en su construcción se encontró que existían dibujos que evocaban algunos conceptos que actuaban como estímulos que "inhibían" el leer o escribir, otros que actuaban como estímulos "desencadenantes", y otros que actuaban como "neutros" para el aprendizaje de la lectoescritura. Eas por ello que la aplicación de las fichas-guías se inicia con la utilización de dibujos considerados como "neutros" o "desencadenantes", descartándose la utilización de dibujos como mamá o papá, porque en muchas de las poblaciones escolares se manejan situaciones familiares especiales que invitan a evitar su utilización.

La autora del método propone como objeto de análisis y discusión, la afirmación de que a partir del análisis de los antecedentes familiares (AF), en asociación con los de Desarrollo (AD) y los académicos (AA), es posible predecir lo que sucederá respecto al rendimiento académico que presentará un estudiante.

Por rendimiento académico (RA) se entenderá el grado de habilidades, destrezas, conocimientos y/o rutinas que debe presentar un estudiante, como consecuencia de la experiencia, de las oportunidades y de la madurez propia de su edad.

Lo anterior indica que cuando alguna de las tres variables ( $\mathrm{AF}, \mathrm{AD}$ o $\mathrm{AA}$ ) es afectada notablemente, se generará un bajo rendimiento académico, facilitando en muchas ocasiones la aparición de los problemas de aprendizaje, PA. 
Es decir, aunque no todo bajo rendimiento es generado por un problema de aprendizaje, sí todo problema de aprendizaje generará un bajo rendimiento.

Por último, se registran las tres cartillas que se han construido como consecuencia de la aplicación de los principios del método MACPA.

\section{Grupos de estudiantes en una institución educativa}

A partir de la asociación que se establece entre los antecedentes familiares (AF), con los de Desarrollo (AD) y los académicos (AA), e involucrando dentro de cada uno de ellos la calidad en las oportunidades, es posible caracterizar por lo menos cuatro grupos de estudiantes dentro de una institución educativa, que activan de manera particular una compleja red de relaciones (que incluyen igualmente a redes previamente constituidas, como la familia y la escuela), y así se ve afectado de manera fundamental el estudiante directamente comprometido.

El primer grupo está conformado por estudiantes conocidos tradicionalmente como los más aventajados del curso, los cuales se caracterizan porque adquieren con relativa facilidad los aprendizajes necesarios y suficientes para su promoción al grado siguiente; como consecuencia, activan una serie de reacciones emocionales entre sus docentes, compañeros y familiares cercanos, que los motivan a seguir siendo los mejores.

El segundo grupo está constituido por los estudiantes que se caracterizan por tener un rendimiento académico regular, sin presentar mayores dificultades para acceder a los conocimientos impartidos por sus docentes; aunque no los adquieren con la misma facilidad que el grupo anterior, generando una serie de reacciones entre sus familiares, docentes y compañeros, los cuales les permiten seguir adelante.
El tercer grupo está conformado por los estudiantes denominados comúnmente como "lentos" en la adquisición de los conocimientos académicos, sin embargo, no presentan características que obstaculizan de manera relevante su tránsito por los diferentes cursos, aunque van accediendo al aprendizaje de manera pausada, haciendo necesario que el maestro y la familia los ayuden, incorporando estrategias complementarias a las que se utilizan de manera regular.

El cuarto grupo está constituido por los estudiantes de los cuales se ocupa este artículo, es decir, por estudiantes que presentan problemas de aprendizaje. Su existencia en un aula de clase obliga a maestros, profesionales de apoyo y familia, a buscar toda clase de estrategias que les permitan seguir adelante con sus estudios.

El número de casos reportados por los docentes de los grados primero y segundo varía entre el $14 \%$ y el $28 \%$; es decir, de un grupo de 35 niños, se reportan entre 5 y 10 niños, los cuales a su vez evidencian dificultades que se ubican entre niveles moderados o graves.

\section{Problemas de aprendizaje}

La definición de los problemas de aprendizaje, PA, se refiere necesariamente a los aprendizajes escolares, por cuanto dentro del cuadro característico que evidencian estos niños, están los aprendizajes típicamente escolares como la lectura, la escritura, las matemáticas, que afectan el rendimiento de las otras áreas (García y González, 2001).

En base a lo anterior y a partir de las características que se evidencian en la población que presenta problemas de aprendizaje, es posible realizar una clasificación teniendo en cuenta las variables edades, antecedentes académicos, rendimiento académico, niveles de comprensión y ejecución, ubicación actual y número de grados repetidos, como lo detallo a continuación. 
Problemas de aprendizaje leve. Se refiere a las conductas académicas que presenta un niño cuando está accediendo al proceso de lectoescritura, en donde se encuentran omisiones, sustituciones, inversiones, separaciones, rotaciones de letras y de palabras. Asimismo, es posible observar vacíos en los aprendizajes que serían propios para su edad, tales como discriminar formas, figuras, colores, tamaños, realizar series, ubicar objetos en el espacio, identificar figuras en un fondo y otras más, referidas al área de percepción visual (Molano, 2006, 2009, 2010).

Para que sea considerado como problema de aprendizaje leve, el niño debe estar cursando por primera vez el grado en el cual se encuentra ubicado, no haber repetido el curso primero, y no tener una edad superior a un año de la esperada para el grado en el cual se encuentra. En general,

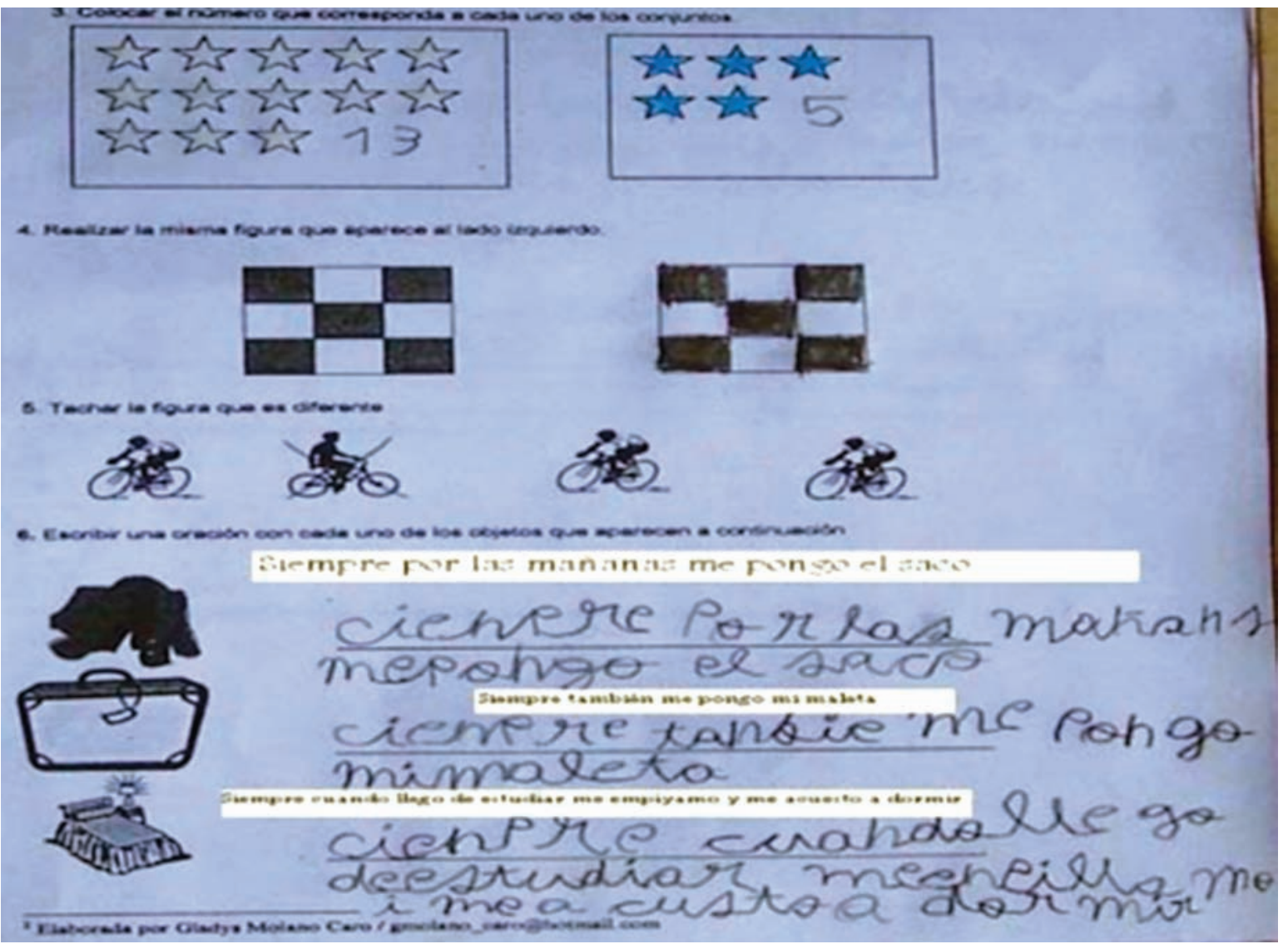

Gráfica 1: Escrito realizado por un niño de 8 años ubicado en el segundo grado de primaria.

los niños que presentan un problema de aprendizaje leve, se encuentran cursando los grados primero o segundo de primaria (ver Gráfica 1).

Problemas de aprendizaje moderado. Comprende al grupo de niños y niñas que en general tiene como antecedente académico la repitencia de los grados primero y segundo, y que presentan problemas básicamente en el área de lectura y escritura, referidos a omisiones, sustituciones, inversiones, contaminaciones, rotaciones de letras, sílabas o palabras, en el momento de leer 
o de escribir de manera espontánea o al tomar dictados (Molano, 2006, 2009, 2010).

Se debe igualmente aclarar, que se trata de dificultades con alta frecuencia que obliga a la intervención de estrategias muy bien seleccionadas para lograr su solución. Los niños que presentan un problema de aprendizaje moderado, en general están con una diferencia de por lo menos dos años en relación con la edad esperada para ser ubicados en los grados primero o segundo. Asimismo, es común encontrar que estos niños no evidencian dificultades referidas al cálculo matemático y resuelven con relativa facilidad los problemas cotidianos de su entorno (ver Gráfica 2).

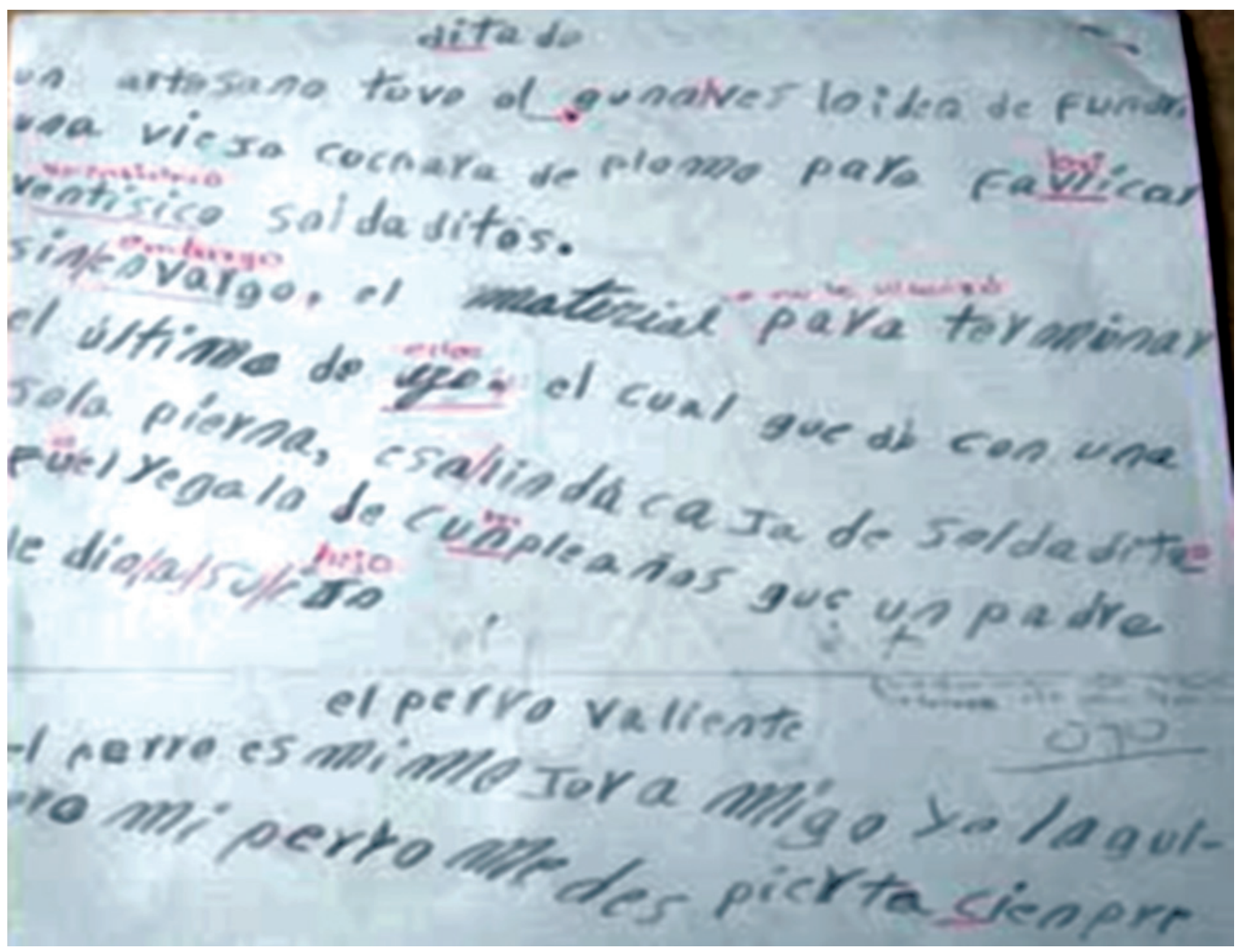

Gráfica 2. Producción escrita de un niño de 9 años en la cual es posible observar errores de omisión, sustitución, entre otros.

Problemas de aprendizaje grave. Denominados así porque a pesar de que el niño tiene una inteligencia "normal", como el caso que aparece expuesto en la Gráfica 3, el grado de afectaciones en su aprendizaje es tan alto, que impide que el niño o la niña escriban, lean, hablen, y/o realicen cálculos matemáticos, de la manera correcta, a pesar de haber estado expuestos a condiciones de aprendizaje relativamente adecuadas (Molano, 2006, 2009, 2010).

Asimismo, la edad que presentan estos niños excede por lo general en más de tres años 
la edad promedio en que deberían estar cursando cada grado y han repetido por lo menos dos veces el grado primero de primaria. Al igual que en los casos anteriores, las dificultades en la lectura y escritura se evidencian en omisiones, sustituciones, agregaciones, inversiones, rotaciones, uniones o separaciones incorrectas, así como dificultades de comprensión y de ejecución en contextos que obligan al análisis.

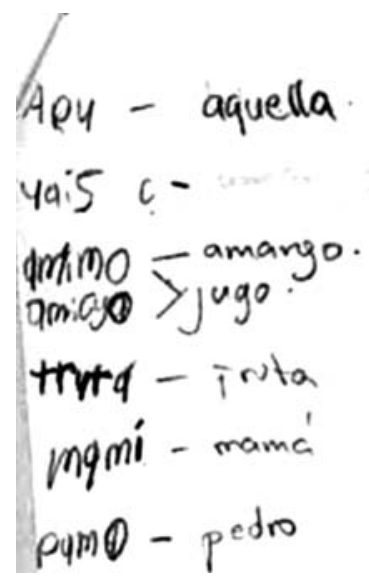

Gráfica 3: Escrito realizado por un niño de 8 años ubicado en el primer grado de primaria.

Los estudiantes con problemas de aprendizaje requieren más tiempo para crear el hábito de dirigir la atención de un estímulo a otro; esto implica una intencionalidad, por lo cual se precisa de una fuerte motivación para mantener su interés. Asimismo, tienen gran dificultad en inhibir o retener su respuesta hasta después de haberse tomado el tiempo de examinar con detalle los aspectos más útiles y/o los componentes más abstractos de los estímulos; a ello se debe la menor calidad de su respuesta y su mayor frecuencia de errores (Luria, 1984).

La mayoría de profesionales que trabajan con problemas de aprendizaje, indican que una de las grandes dificultades en su abordaje, es el realizar un diagnóstico de manera tardía. Básicamente, se podría decir que existe una relación directamente proporcional respecto al tiempo en que se realiza el diagnóstico, al tiempo empleado en la solución que se debe enfrentar; es decir, entre más pronto se diagnostica, más pronto se podrá hallar la solución.

Para Hammill (1993) y García y González (2001), la definición de problemas de aprendizaje da cuenta de una tendencia que está presente a lo largo del ciclo vital y que requiere de respuestas selectivas en el marco de la escuela obligatoria, que involucra el concepto de necesidades educativas especiales, y que demanda la adopción de un currículo especial para los estudiantes con estas necesidades.

Asimismo, Hammill (1993), al realizar un estudio respecto al término de problemas de aprendizaje, concluye indicando que uno de los aspectos en los cuales se halló coincidencias con otros autores recientes, fue el incorporar lo referido al bajo rendimiento que presenta este grupo de estudiantes.

Los estudios realizados por Mercer (1987), concluyen indicando que los estudiantes con problemas de aprendizaje presentan una "discrepancia" entre las expectativas del rendimiento y lo que evidencian en el salón de clase.

El DSM-IV, indica que el bajo nivel de logros alcanzado por un niño que presenta problemas de aprendizaje interfiere significativamente en el rendimiento académico o en las actividades de la vida cotidiana, especialmente en aquellas que exigen la utilización de las habilidades de lectura, escritura o cálculo (García \& González, 2001).

Lo anterior indica que leer y escribir no son aprendizajes que surgen únicamente como consecuencia directa de la enseñanza ejercida por un adulto o personas que hayan tenido el interés de realizarlo. Se podría decir que en cada uno de esos procesos, convergen más de los que suponemos, la afectividad y la calidad de la exposición a oportunidades y a experiencias.

Frente a condiciones normales, todos los niños y niñas tienen la capacidad para adquirir cualquier aprendizaje e ir desarrollando las diferentes habilidades que favorezcan su desempeño, 
incluyendo la lectura, la escritura y las matemáticas; sin embargo, esos aprendizajes y habilidades que deberían ser comunes a la población escolar, requieren de la participación/asociación en proporciones casi idénticas de los aspectos cognitivos, psicomotores, comunicativos y afectivos, siendo éste último, a juicio de la autora, el que potencializa los otros tres aspectos.

En lo observado, se encontró que el aspecto afectivo es el que facilita que ocurra de manera sencilla tanto el aprendizaje de la lectura como el de la escritura; el cual al haber estado atendido de manera adecuada, no se constituye en un obstáculo que frene, altere o inhiba los potenciales de aprendizaje que deben estar presentes para acceder de manera adecuada a todos los conocimientos.

Por el contrario, cuando ello no ocurre así, cuando la parte afectiva no ha sido atendida de manera adecuada, se presentan problemas de aprendizaje, en algunos casos, y de comportamiento en otros. A veces se hacen presentes diferentes trastornos de conducta escolar como el no querer permanecer en el salón de clase, agredir a los compañeros, no seguir instrucciones de los adultos, dificultades al interactuar no solo en la infancia sino en la edad adulta con sus pares, dificultad en el reconocimiento de la autoridad y la afectación en la autoimagen, entre otras dificultades derivadas.

En consecuencia, la presencia en el aula de un grupo de niños que presentan esta clase de problemas debería llevar a que el docente reflexione sobre los siguientes temas:

Existe un tiempo para aprender. Se debe tener en cuenta que aunque todos los niños necesitan de los mismos procesos para aprender, no todos han estado expuestos a situaciones ricas en estimulación, lo cual genera en algunos de ellos, ritmos lentos de aprendizaje, inmadurez escolar y problemas de aprendizaje, requiriendo un mayor tiempo de lo esperado para adquirir los diferentes conceptos (Molano, 2006, 2009, 2010).

La respuesta de los organismos. No todos los organismos responden de la misma manera a los diferentes estímulos; algunos niños requieren de más tiempo de exposición a experiencias de mayor estimulación para poder adquirir un conocimiento. Es por ello, que en ocasiones se debe "exagerar" en la realización de actividades, por ejemplo, algunos niños con unas pocas actividades aprenden conceptos como adelante, atrás, arriba, abajo, etc.; otros niños requieren que se les brinden condiciones de mucha imaginación y creatividad por parte del adulto para que puedan comprender estos conceptos.

La aprobación del adulto. Hay niños que requieren continuamente de la aprobación del adulto para tener la "seguridad" de que están haciendo las cosas bien, de esta manera, es conveniente "reforzar" el comportamiento de los niños que presentan más inseguridad con frases como: ¡tú puedes!, ¡muy bien!, ¡te felicito!, ¡qué maravilla!, ¡mira, cómo te quedó de lindo!, ¡estás progresando!, y otras más que se les puede compartir.

Las condiciones ambientales. Un gran número de niños de las instituciones educativas no cuentan con las mejores condiciones para poder estudiar en sus viviendas, no cuentan ni con los espacios ni con los materiales adecuados. En ocasiones, tampoco cuentan con un adulto que les oriente en la realización de sus tareas; por lo tanto, se requiere que la escuela les entregue o proporcione todas las situaciones que les estimulen a seguir aprendiendo.

Las expectativas generadas. Es común dar por hecho que un niño que ingresa al grado de transición, trae en su haber una serie de aprendizajes; sin embargo, la realidad es otra. Muchos de ellos desconocen formas, colores, tamaños, posiciones espaciales, etc.; su nivel de comprensión refleja que no han sido expuestos a situaciones adecuadas de estimulación. Se requiere, por lo tanto, que la escuela les proporcione con estas situaciones, incrementando los niveles de estimulación y de tiempo de exposición a las mismas.

La responsabilidad asumida. Aunque todos los docentes asumen la responsabilidad de formar un grupo de estudiantes enseñándoles conceptos, ayudándoles a desarrollar una serie de 
habilidades, generando en este grupo, rutinas y hábitos, despertando intereses por adquirir nuevos conocimientos, etc.; no siempre puede ser asumida con la tranquilidad y la serenidad que se desearía. Básicamente se encuentran una serie de obstáculos en el camino, como los que se han venido exponiendo, que les impiden cumplir con ese propósito.

La dificultad de poder alcanzar el reto de sacar adelante a todos los estudiantes, no debe generar como reacción obvia el sentirse derrotado. Todo lo contrario. El docente debe estimular la creatividad que permita generar el impulso de lograr que todos los estudiantes obtengan el aprendizaje más adecuado posible, comprendiendo asimismo, que al no haber elegido los niños esa condición, necesitan del docente "sensato" para salir adelante.

El hacer obvio lo que es obvio. Son muchas las situaciones de vida en donde es preciso hacer caer en cuenta a una persona de que un hecho está sucediendo; ello se debe básicamente a que no todos le prestamos el mismo interés a las cosas que se observan. Por ello, en ocasiones, se debe hacer caer en cuenta a los niños que están observando tal o cual situación para que incorporen ese conocimiento.

\section{Condiciones que favorecen la aparición de problemas de aprendizaje en el aula}

El trazo en la enseñanza de la escritura y la lectura. Una de las acciones que son generadas comúnmente por los adultos cuando les enseñan a los niños, radica en que no realizan el trazo de las letras de manera adecuada y con suficiente distinción entre el trazo de una y otra letra. Por ejemplo: globo o sapo, es posible que el niño realice la lectura tal como se pretende, sin embargo, también es posible que al hacer el trazo de la "l" o de la "b" o de la "s", no se haya realizado con suficiente claridad o precisión, y sea entendida por el niño la "l", por ejemplo, como una "i" o como una "e". Así como en el caso de la "b", podría ser asumida como una "o" o una "p", e inclusive como una " $d$ " $y$, en el caso de la "s" sea leída como una "r" o una "v".

El nombre de las letras. Cuando se da el nombre a las letras $\mathbf{g} \mathbf{b} \mathbf{r} \mathbf{s} \mathbf{t} \mathbf{l} \mathbf{j}$, éstas podrán ser comprendidas por la mayoría de niños según el nombre que se les ha asignado, y tomarán su sonido dentro del contexto de una palabra según corresponda; sin embargo, también es posible que cuando el niño trate de realizar la lectura dentro de una palabra, asuma el nombre que se la ha asignado a la letra. Por ejemplo:

\section{CASA entendida SESA como}

Esto debido a que la letra "c" toma el valor o el significado que se le asigna en el abecedario, es decir, "se". Así ocurre con muchas palabras, por ejemplo, cuando se le dicta a un niño la palabra "es", puede leer y escribir "ese" o "eses".

Proceso de seriación. No se debe perder de vista que la escritura y la lectura son una serie de códigos arbitrarios que no tienen ningún parecido con el objeto que representan, es decir, el niño debe realizar procesos de asociación complejos para poder comprender que la palabra "teléfono" identifica un concepto y que dependiendo de la ordenación de las letras, toma diferentes significados. Por ejemplo, las letras “c" “a" "s" “a”, pueden ser leídas según el orden que asumen como "casa" o como "saca", lo cual otorga un significado bien diferente a cada una de ellas.

Proceso de asociación. A algunos niños le es difícil asociar las partes con el todo, es decir, presentan un alto grado de dificultad al realizar la integración entre las partes y el significado que asumen cuando se integran, quedándoles más fácil otorgarle valor al "todo", para luego com- 
prender los elementos que la constituyen. Así por ejemplo, si se le enseña al niño:

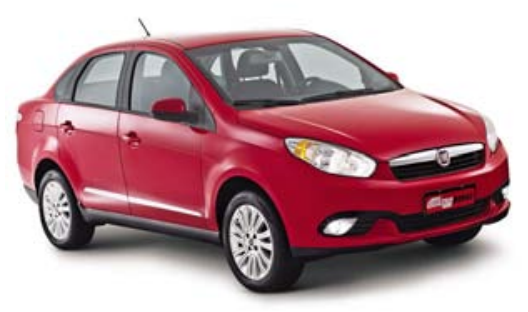

Entenderá con mayor facilidad, que si se le enseña la "c" con la "a", "ca"; la "rr" con la "o" "rro".

¿Pero no sabe ni las vocales! Esta exclamación es común escucharla. Sin embargo, se debe revisar si realmente el niño no sabe las vocales o lo que pasa es que las "aprendió" como se las enseñaron, es decir, como la serie “a, e, i, o, u”, generando en el niño la "certeza" de que esa es la forma de escribirlas. Es decir, cuando el adulto trata de verificar si realmente conoce las vocales y le dicta “e” como la primera vocal, el niño escribe la “a", pensando seguramente que lo está haciendo bien.

En este caso, sería conveniente hacerle caer en cuenta al niño la asociación entre el sonido, la forma y el nombre de cada una de las vocales. $\mathrm{Al}$ respecto se aconseja:

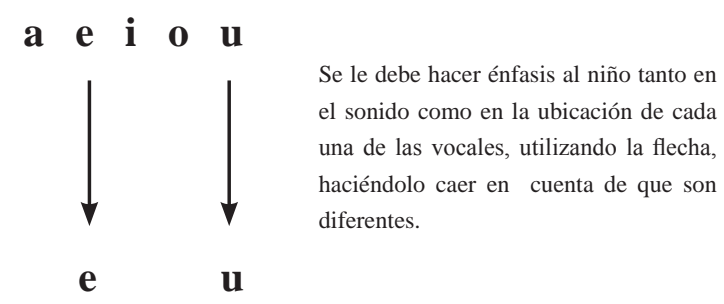

La letra cursiva y la script. Cuando el niño no ha cimentado de manera suficiente el aprendizaje de la lectoescritura con alguna de las dos letras, y se le "obliga" a adoptar una diferente de la que se halla en proceso, se genera una "distorsión" en el trazo que afecta de manera relevante su producción escrita. Por ello se aconseja que se debe "respetar" la letra con la cual el niño ha iniciado el proceso, independientemente de cuál haya sido la letra.

Problemas del método. En ocasiones se pueden observar casos "mixtos" de origen de dificultad. Por ejemplo, niños que distorsionan el trazo porque la persona que les enseñó no lo realizaba de la manera más precisa posible, así como al enseñarle lo hicieron utilizando el método alfabético, fonético o silábico. Inclusive, es posible encontrar a niños que "aprendieron" con el método propio de sus padres, que resulta generalmente de una "mezcla" entre los tres anteriores y la propia jerga que utilizan los padres, por ejemplo, no dicen "abuelos" sino "aguelos", no "fiesta" sino "jiesta".

Los llamados grupos consonánticos. En general, los docentes de los grados de transición o de primero, inician el proceso lectoescrito enseñando las palabras "mamá" o "papá” y todas las que de allí derivan, continúan luego con las palabras que contienen las letras " $\mathrm{s}$ ", "l", " $\mathrm{t}$ ", etc.; $\mathrm{y}$ asumen que las últimas deben ser las palabras que contengan las letras como la " $y$ " o la "rr". Sin embargo, en la aplicación/adecuación del método, se encontró que los niños aprendían con prontitud y agrado las palabras como "yo", "carro", "mariposa", "teléfono", "pan”, etc.

\section{El método MACPA}

Antes de describir el método, es importante tener en cuenta que leer y escribir no son aprendizajes que se adquieran en un solo momento y como consecuencia de unas pocas sesiones de enseñanza. Por el contrario, solo se adquieren mediante la exposición a condiciones adecuadas en armonía con factores fisiológicos y psicológicos. La exposición al medio, en condiciones afectivas adecuadas, favorece a que los niños o niñas adquieran, hagan uso y desarrollen los aprendizajes. Solo así se alcanzarán buenos lectores y escritores. 
La lectura es una actividad compleja que involucra procesos perceptivos, léxicos, sintácticos y semánticos. A partir de los procesos perceptivos se extrae información de las formas de las letras y palabras, la cual permanece en la memoria icónica por periodos cortos de tiempo. Se requiere asignarle significado al reconocimiento de las palabras para que se pueda avanzar hacia su comprensión (Ramos, 2000).

Los procesos léxicos o de reconocimiento de palabras, son los que nos permiten acceder al significado de las mismas. Por su parte, los procesos sintácticos colaboran en identificar las distintas partes de la oración y su valor relativo para acceder correctamente al significado del mensaje. Por último, se encuentra el proceso semántico que es el que nos permite acceder a la comprensión eficaz de un texto (Ramos, 2000).

En la escritura se encuentran presentes cuatro procesos cognitivos: la planificación del mensaje, la construcción de las estructuras sintácticas, la selección adecuada de palabras y los procesos motores (Ramos, 2000).

Aunque en principio se asume que la escritura es un proceso cognitivo complejo, la emoción, y en especial, el afecto, ingresan para darles sentido. El protagonismo que asume la afectividad en su consolidación, se ve reflejado en las etapas de adquisición, uso y desarrollo de las palabras (Molano, 2010).

En las etapas de adquisición y uso de la lectura y la escritura, el afecto se articula con la cognición para dar sentido, asociar, relacionar los conocimientos, las experiencias, las sensaciones y las emociones con los códigos. En la etapa del desarrollo, son las oportunidades y la exposición a condiciones adecuadas, las que permiten desarrollar estas dos habilidades (Molano 2010).

Si una persona pretende establecer que ha comprendido un texto, el reto será escribir sobre él, porque la escritura orienta e interroga una y otra vez, favoreciendo el diálogo y la discusión con nosotros mismos, exigiéndonos a retomar el texto. La escritura nos brinda la oportunidad de problematizar el acto mismo de escribir. Es por ello que se trata de un proceso complejo que demanda la intervención de los procesos de pensamiento y del afecto y emoción para su consolidación (Rincón, 2009; Molano, 2010).

Con estos considerandos y con el camino que fueron señalando más de 50 niños, niñas y jóvenes con los cuales se fueron validando una a una las diferentes fichas-guías, se dio inicio a la construcción del método que tuvo en cuenta, además de lo ya descrito, lo siguiente:

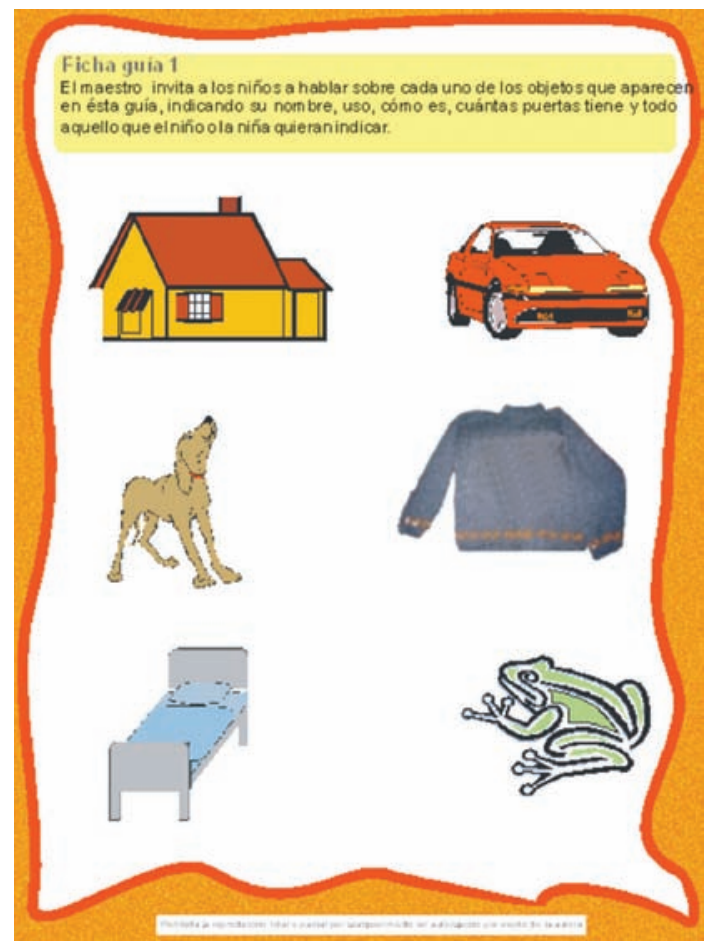

a) El método MACPA está constituido por fichas-guías que utilizan como referentes de motivación animales, objetos y/o situaciones que fueron seleccionadas, luego de la observación que hizo la autora durante tres años continuos de intervención con niños y niñas que presentaban problemas de aprendizaje.

b) Durante la construcción del método, se encontró que existen palabras que encierran un alto grado de afecto para los niños, generándoles diferentes respuestas que van desde la inhibición, la activación o inclusive actúan como 
estímulos neutros; ello es, no representan ni una carga afectiva positiva, ni una carga afectiva negativa.

De esta manera, como se puede observar más adelante, en las primeras fichas-guías se utilizan dibujos y palabras como "casa" que pueden ser consideradas como estímulos neutros, así como dibujos y palabras como "carro", "perro" o "mariposa”, palabras que actúan como estímulos desencadenantes. Se evita la utilización de palabras como "mamá" o "papá", porque se observó en muchos de los niños reacciones emocionales negativas, que produjeron inhibición al realizar la lectura y la escritura.

c) Asimismo, el grupo de niños que presentan problemas de aprendizaje ya iniciaron su proceso de lectura y escritura y el manejo de las matemáticas, por lo que la elaboración de estas fichas-guías parte de ello, dejando de lado el error, a juicio de la autora, de reiniciar el proceso con los métodos tradicionales.

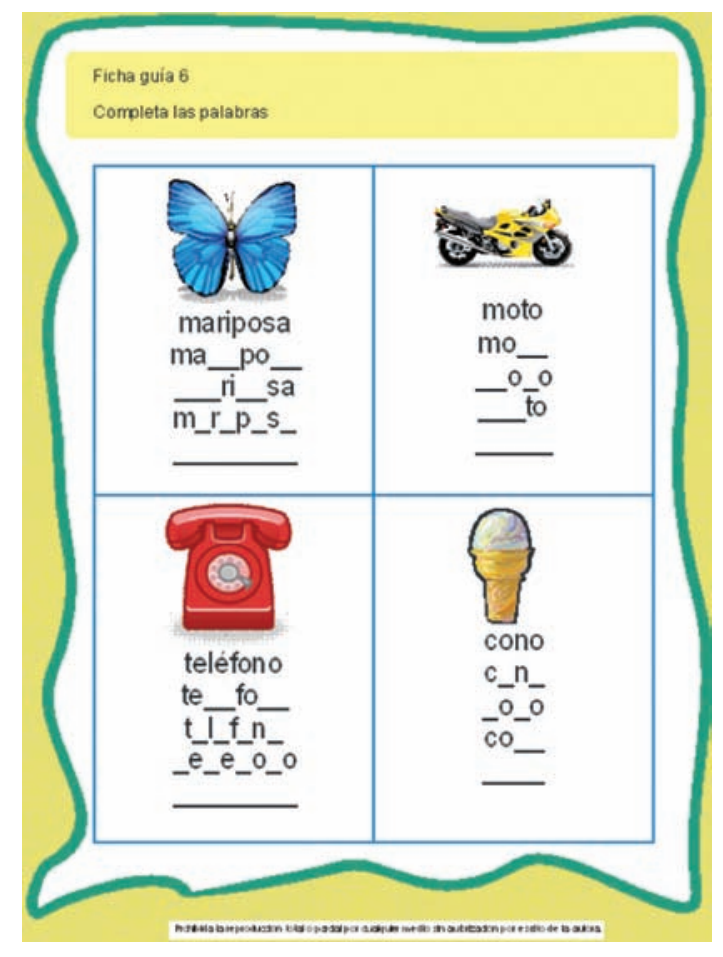

d) Los niños que presentan problemas de aprendizaje se enfrentan continuamente a la frustración y el rechazo, tanto del adulto como de sus mismos compañeros, porque no aprenden de la misma manera. Partiendo de esta realidad, cada una de las fichas-guías fue construida evitando al máximo hacer caer al niño o a la niña en errores, conduciéndolo a un aprendizaje con niveles altos de motivación e interés.

e) Se utiliza la observación, en lo posible, de elementos que estén fácilmente en contacto con el niño y la niña, como principal herramienta, para que vayan accediendo al conocimiento, apoyándose fundamentalmente de láminas. Esto explica el por qué de ellas en cada una de las guías.

f) Las fichas-guías han sido construidas basándose en los principios de la pedagogía activa, sin embargo, la secuencia en su presentación y elaboración obedece a la observación de la autora del método sobre la motivación y el interés que presentan los niños en los diferentes temas que van aprendiendo. Ello explica su presentación, que se realiza con dificultad creciente respecto a los conocimientos que deben ir adquiriendo los niños.

g) En cada una de las fichas-guías se conduce al niño o a la niña al conocimiento, haciéndolos caer en cuenta en la lógica de cada una de las respuestas que deben ir dando; para ello se recurre al sentido común que está presente en todas las personas. Aquí toma sentido el hacer obvio, lo que es obvio.

h) Las fichas-guías fueron creadas por orden de complejidad, de allí que puedan ser utilizadas para los niños que se encuentran en los grados primero, segundo o tercero.

i) Las fichas-guías desarrollan la habilidad de prever, de sacar conclusiones, de inferir, de resolver problemas, de dar una respuesta que indique que se ha comprendido lo aprendido. Todo ello a partir de preguntas sencillas que realiza el docente, y que hacen que los niños se apropien de cada aspecto trabajado allí. 


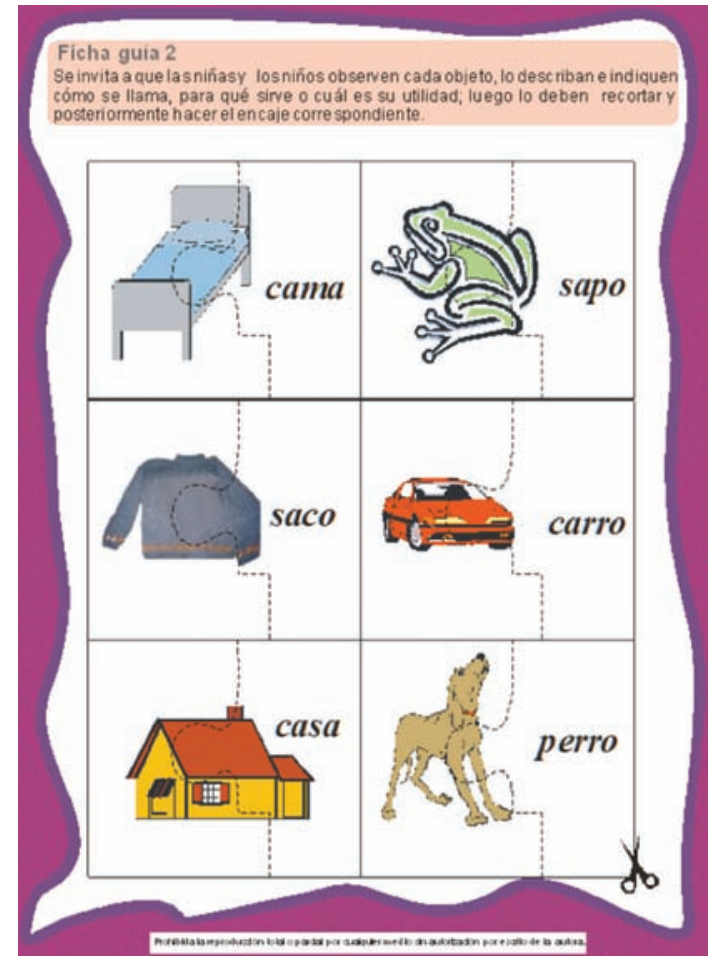

j) Cada ficha-guía evita estar saturada de estímulos visuales, porque se ha encontrado que la mayoría de estos niños tiene dificultades de figura-fondo.

k) A través del método, el niño realiza ejercicios de recortar, encajar, asociar, construir frases, oraciones, párrafos e historias que van surgiendo con espontaneidad de los mismos niños.

1) El tiempo de cada una de las sesiones en lo posible no deberá superar las dos horas.

m) Las condiciones ambientales para su aplicación deberán ser las mismas que se tienen en cuenta para emprender un trabajo escolar, esto es, deberá existir una luz adecuada, un pupitre y un asiento que le permita al niño encontrarse cómodo, así como no deberán existir ruidos externos que afecten la concentración del niño.

n) Por último, recuerde que el niño con problemas de aprendizaje no eligió esta condición. Por lo tanto, requiere de todo el apoyo y com- prensión que podamos darle para que salga adelante. Entre esos apoyos están, además de los expuestos, el evitar al máximo utilizar frases descalificadoras como "yo ya te había indicado", "es que no pones cuidado", "tú no quieres aprender", "le tengo que decir a tu mamá que no quieres aprender", "ipor qué los demás niños sí aprenden y tú no?”, “si yo le enseñé a tus hermanos y todos aprendieron a leer", etc.

A partir del método MACPA se han elaborado tres productos o cartillas denominadas: "Mi mascota Mac", la cual se utiliza para niños, niñas y jóvenes con extraedad escolar y alta repitencia escolar; "Mi mascota Mac me acompaña a leer y a escribir" diseñada para prevenir o intervenir de manera oportuna el aprendizaje de aquellos estudiantes que no adquieren al mismo ritmo que sus compañeros de grado primero o segundo la lectura y la escritura; y "Mi mascota Mac me ayuda a comprender" cierra el proceso para los dos grupos anteriores.

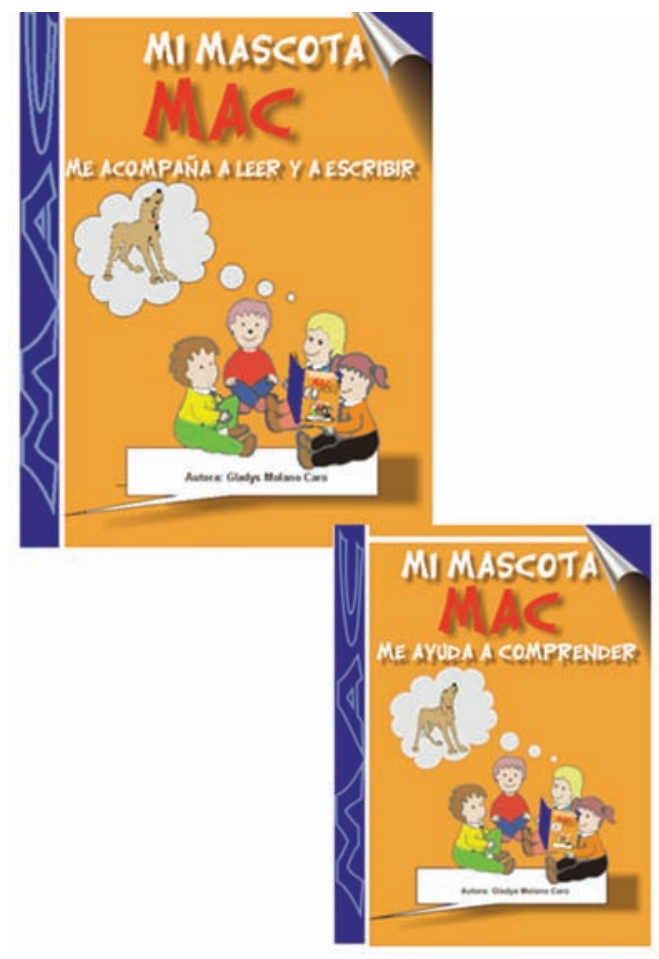




\section{A manera de conclusión}

El método MACPA fue construido al articular un pensar, un sentir y un hacer. Coloca al afecto como antecesor de conocimientos sólidos, como deben ser la lectura y la escritura. La selección del material que constituye las tres cartillas que hasta el momento se han elaborado, fueron construidas en el diario hacer con los niños, niñas y jóvenes. Todo el material fue validado a través de su uso en innumerables sesiones de trabajo. De ahí el éxito que hasta el momento ha tenido.

El afecto puede influir positiva o negativamente en el proceso de enseñanza-aprendizaje debido a la contundencia de sus efectos en la persona. Es decir, el afecto crea una adhesión especial en el aprendizaje cuando éste procede de personas que representan autoridad para los niños; de tal manera que la impronta generada, difícilmente será reemplazada y/o borrada. Entre mayor es la carga afectiva, mayor será la adhesión, y mayor será la dificultad de solucionar un problema de aprendizaje que se haya generado como consecuencia de la aplicación incorrecta del método empleado para enseñar a leer y a escribir.

Lo anterior también indica que la asociación entre la aplicación incorrecta del método que se utiliza para enseñar a leer y escribir y el afecto, desplegado por la persona que la ejecuta, puede determinar el fracaso académico de un estudiante. Asimismo, la utilización correcta del método asociado con el afecto, determina el éxito a través de aprendizajes sólidos en áreas fundamentales como lo son la lectura y la escritura.

Por último, la aplicación del método MACPA es experimentar la alegría que se siente cuando un estudiante manifiesta que ya sabe leer y escribir. Esto va más allá de toda reflexión posible: concreta el ser docente.

\section{Bibliografía}

Bautista, R.

1993. Necesidades Educativas Especiales. Madrid: Ediciones Aljibe.

Bravo, L.

1995. Lenguaje y dislexias: Enfoque cognitivo del retardo lector, 2da edición. Chile: Universidad Católica de Chile.

García Vidal, J., \& González, D.

2001. Dificultades de Aprendizaje e Intervención Psicopedagógica. Teorías, Evaluación y Tratamiento. Vol. 1. Madrid: EOS.

Grieve, J.

1995. Neuropsicología: Evaluación de la percepción y de la cognición._Bogotá: Editorial Médica Panamericana.

Hammill, D. D.

1993. A brief look at the learning disabilities movement in the United States. Journal of Learning Disabilities, 26, 295-310.

Luria, A. R.

1984. Atención y Memoria. Barcelona: Martínez Roca.

Mercer, C.

1991. Dificultades de Aprendizaje. Barcelona: CEAC.

Molano, G.

2006. Cinco concepciones erróneas en el abordaje de la lectura y la escritura. Revista Educación y Cultura, 71, 71-75.

2009. Tres herramientas pedagógicas para evitar la deserción escolar. Revista Educación Hoy, 180.

2010. Gocemos de la lectura y la escritura con mi mascota Mac, ciclos uno y dos. Bogotá: IDEP.

Pichot, P. (Coord.).

1994. DSM-IV Manual diagnóstico y estadístico de los trastornos mentales. Barcelona: MASSON, S.A.

Ramos, J. L.

2000. Procesos de lectura y escritura: descripción, evaluación e intervención. Venezuela: E.O. E.P., Facultad de Educación (UEX). 